\title{
METHODS OF IMPREGNATING NEUROFIBRILLAR SUBSTANCE ON SLIDES AFTER IMBEDDING
}

\author{
BY \\ PETER MIHÁLIK \\ From the Department of Anatomy, University of the Witwatersrand, Johannesburg
}

This paper describes three new methods for impregnating neurofibrillar substance in sections prepared from imbedded material and then mounted on slides. The first two methods are simpler than any previous slide methods for, after the silver bath, the usual reduction is left out and the silver is replaced directly by gold. All these methods can be modified to some extent for selective impregnation of connective tissue fibres (Fig. 4) simply by adding two drops of concentrated ammonia per $100 \mathrm{ml}$. to the colloidal silver bath, but a discussion of the impregnation of the connective tissue fibres is not included in this paper.

\section{Direct Albumen (Silver-Gold) Method}

By using 0.2 to $0.5 \%$ egg albumen and $2 \%$ silver nitrate in aqueous solution (a colloidal silver bath) it is possible to impregnate the neurofibrillar substance of the central nervous system on slides of imbedded and sectioned materials fixed in a variety of ways. The slides are kept in the silver colloid bath for five days at $37^{\circ} \mathrm{C}$. or for eight days at room temperature, then gilded for 10 minutes by dropping $0.2 \%$ aqueous gold chloride solution onto the slides, and subsequently reduced in a $0.2 \%$ aqueous oxalic acid solution for an hour. Between the steps of the technique, especially between the silver colloid and gold, the slides have to be well washed with distilled water.

This method is extremely simple : over- or underimpregnation or reduction is impossible; the results are selective, free of precipitate, and constant; it works even after decalcification of the material to be impregnated by trichloro-acetic acid.

Fixation.-Fix in $10 \%$ chloral hydrate, according to Cajal, for 24 hours. Wash quickly in distilled water, and then immerse in $96 \%$ or absolute alcohol with 8 drops of concentrated ammonia per $100 \mathrm{ml}$. for another day. Then dehydrate in absolute alcohol and imbed.
Fix in Heidenhain's "Susa" mixture for 24 hours. Wash in several changes of $80 \%$ alcohol, then in $90 \%$ and absolute alcohol, etc.

Decalcification in $5 \%$ trichloro-acetic acid solution for as long as a fortnight directly after fixation alters but little the affinity of the neurofibrillar substance for the colloidal silver and gold. For example, excellent impregnation was obtained in the brain and spinal cord of a guinea-pig when these parts were left in situ with the vertebral column and skull opened and the whole decalcified in a $5 \%$ trichloro-acetic acid bath for 14 days (Figs. 2, 3, 4).

Fixation by perfusion is always superior, especially in the central nervous system where the lipoids hinder the penetration of the fixatives to a greater extent than elsewhere. Perfusion is not essential, however, if the block of tissue is not thicker than $4 \mathrm{~mm}$. Perfusion with "Susa" should be preceded by immersion in some physiological salt solution, because "Susa" clots the blood more rapidly than the other two fixatives, and so blocks the vessels more easily.

Several other fixatives have been tried, but they are not as good as the three already described. Formol alone, or fixatives containing chromates or osmic acid, are inapplicable.

Imbedding.- Imbedding can be done in paraffin or in celloidin paraffin (parlodion). The latter is superior, because shrinkage is less.

Cutting-For neurofibrillary structures in the cell (perikaryon) the sections should be from 4 to $6 \mu$, for fibres and synapses from 7 to $12 \mu$ or even up to $25 \mu$ if so desired.

The slides are coated with (Mayer's) glycerin-albumen and dried in a flame in the usual way. This film is important, not only to prevent detachment of the thicker sections, but it also insulates, eliminating the chemical effect and possible electrical charge of the glass which may interfere with impregnation. The sodium salicylate or the thymol content of glycerin-albumen solution does not alter the impregnation.

It may be noted that good results have been obtained on high quality glass slides after mounting with distilled water alone with thin sections (below $6 \mu$ ) which are less liable to become detached.

Photomicrographs of impregnated sections do not reproduce all the finer details of the sections (e.g. neurofibrils in the perikaryon, etc.). This deficiency is particularly evident in these plates because the sections have a reddish violet tone which does not contrast well with the grey-black of the finer fibres or neurofibrils especially when $15 \mu$ sections are photographed.

All the sections were made from parlodion-paraffin imbedded materials. 


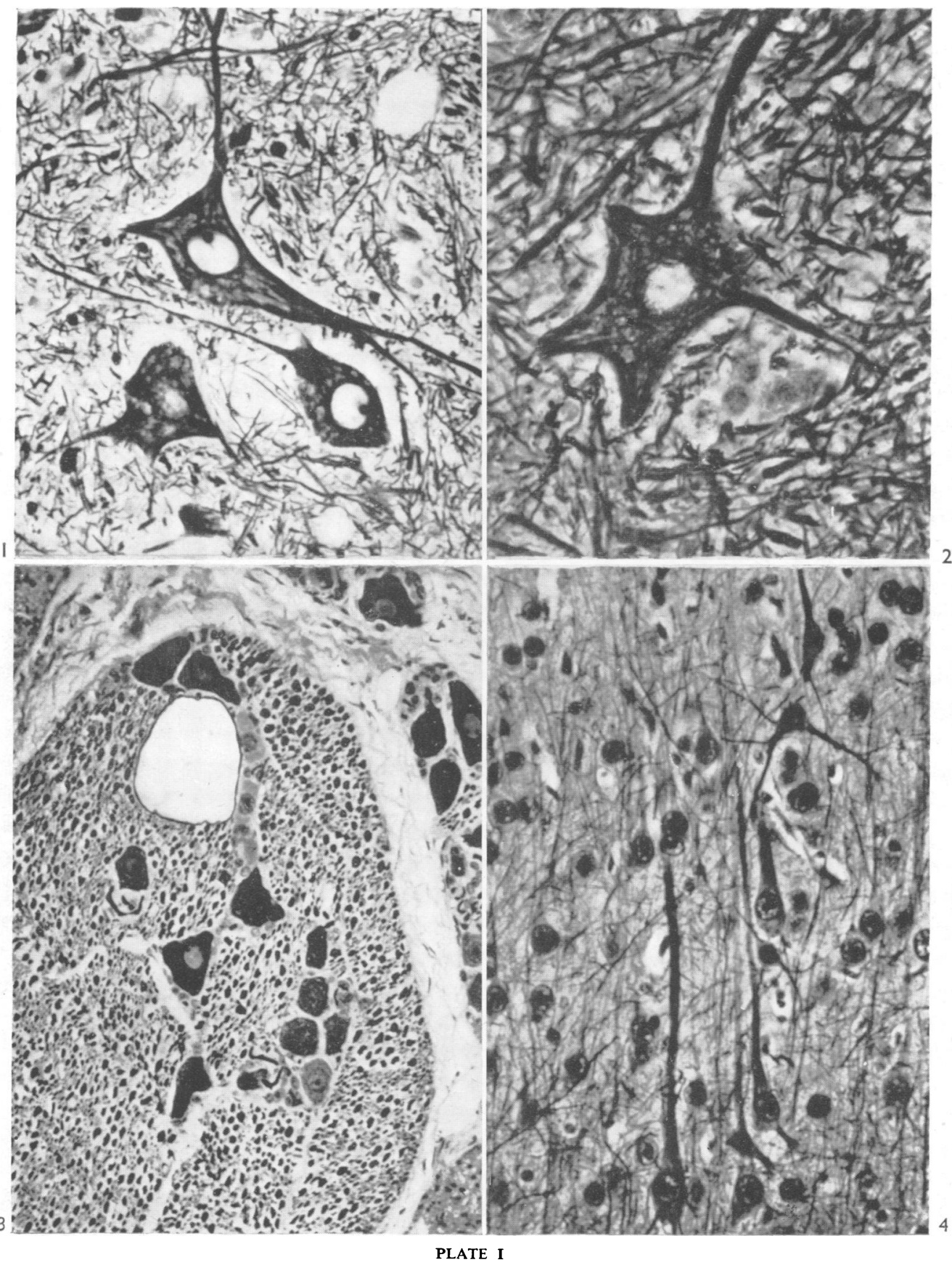

FIG. 1.-Motor cells from the spinal cord of an adult rabbit. Fixed in ammoniacal alcohol $(5 \mu)$. Impregnation in an $0.2 \%$ albumen $+2 \% \mathrm{AgNO}_{3}$ bath five days at $37^{\circ} \mathrm{C}$. Direct albumen method. $(\times 620$.

FIG. 2.-Motor cell from the spinal cord of a young guinea-pig $(7 \mu)$. Fixed and decalcified as in Fig. 5. Impregnation in an $0.2 \%$ albumen $+2 \% \mathrm{AgNO}_{3}$ bath for five days at $37^{\circ} \mathrm{C}$. Direct albumen method. $(\times 800$.

FIG. 3.-Trigeminal nerve near to the ganglion. Material, etc., as in Fig. $2 . \quad(\times 250$.

Fig. 4.-Pyramidal cells, motor cortex. Material, etc., as in Fig. 3. (× 330.) 


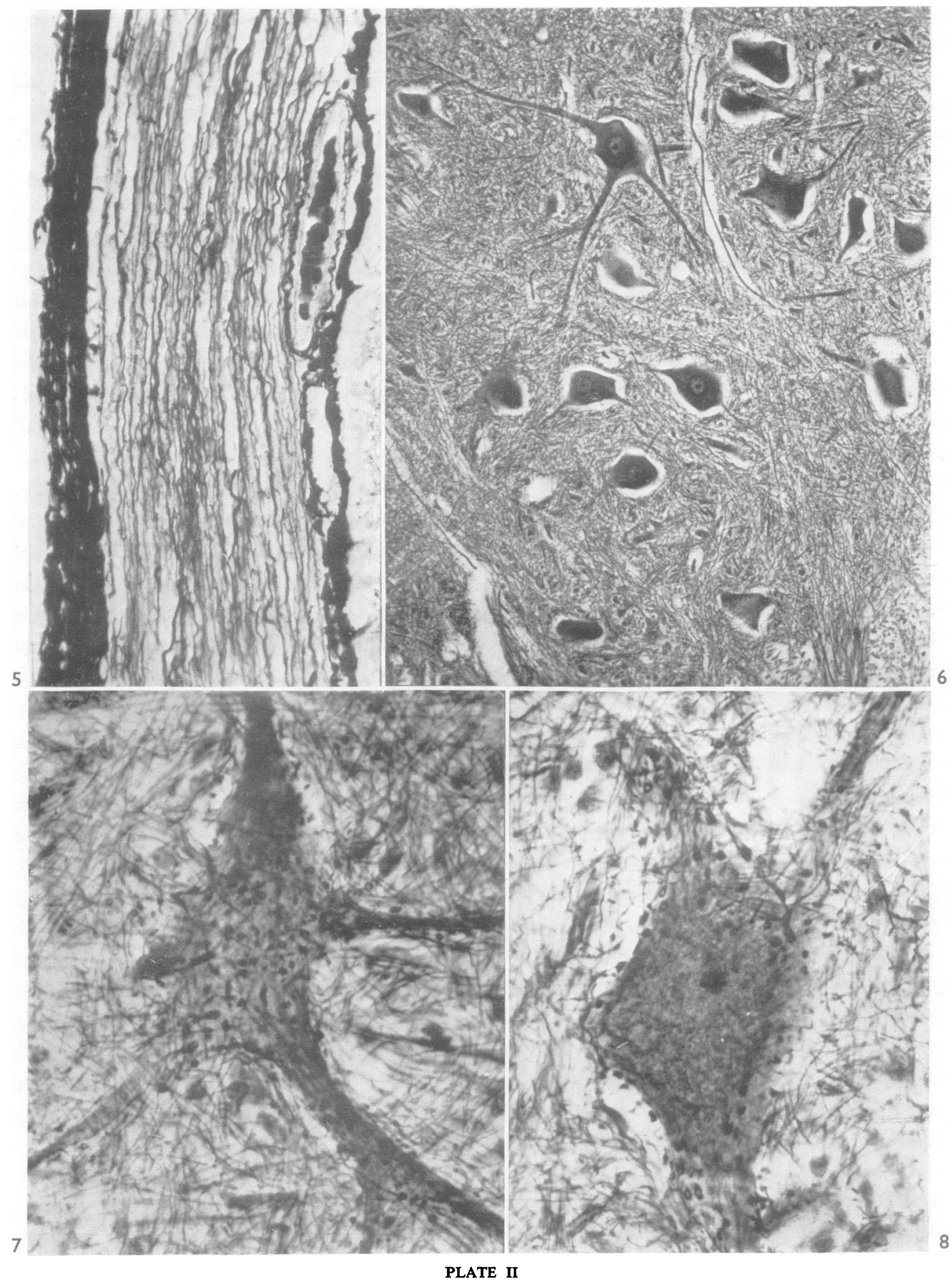

Fig. 5.-Posterior root of a spinal nerve of a young guinea-pig. Fixed in "Susa" in situ together with the vertebral column. Decalcified 14 days in $5 \%$ trichloro-acetic acid $(7 \mu)$ and impregnated by direct albumen method. Only the connective tissue fibres are impregnated because of the $\mathrm{NH}_{3}$ content of the bath. $(\times 450$.

Fig. 6.- Neurocytes from the anterior horn of the spinal cord of an adult cat. Fixed in a $10 \%$ chloral hydrate by transfusion, followed with ammoniacal alcoholic treatment $(15 \mu)$ and impregnated by direct albumen method. $(\times 140$.)

Figs. 7 and 8.-End clubs on neurocytes from the spinal cord of an adult cat. Fixation the same as in Fig. $6(12 \mu)$. Impregnated by direct albumen method. ( $\times 620$.) 


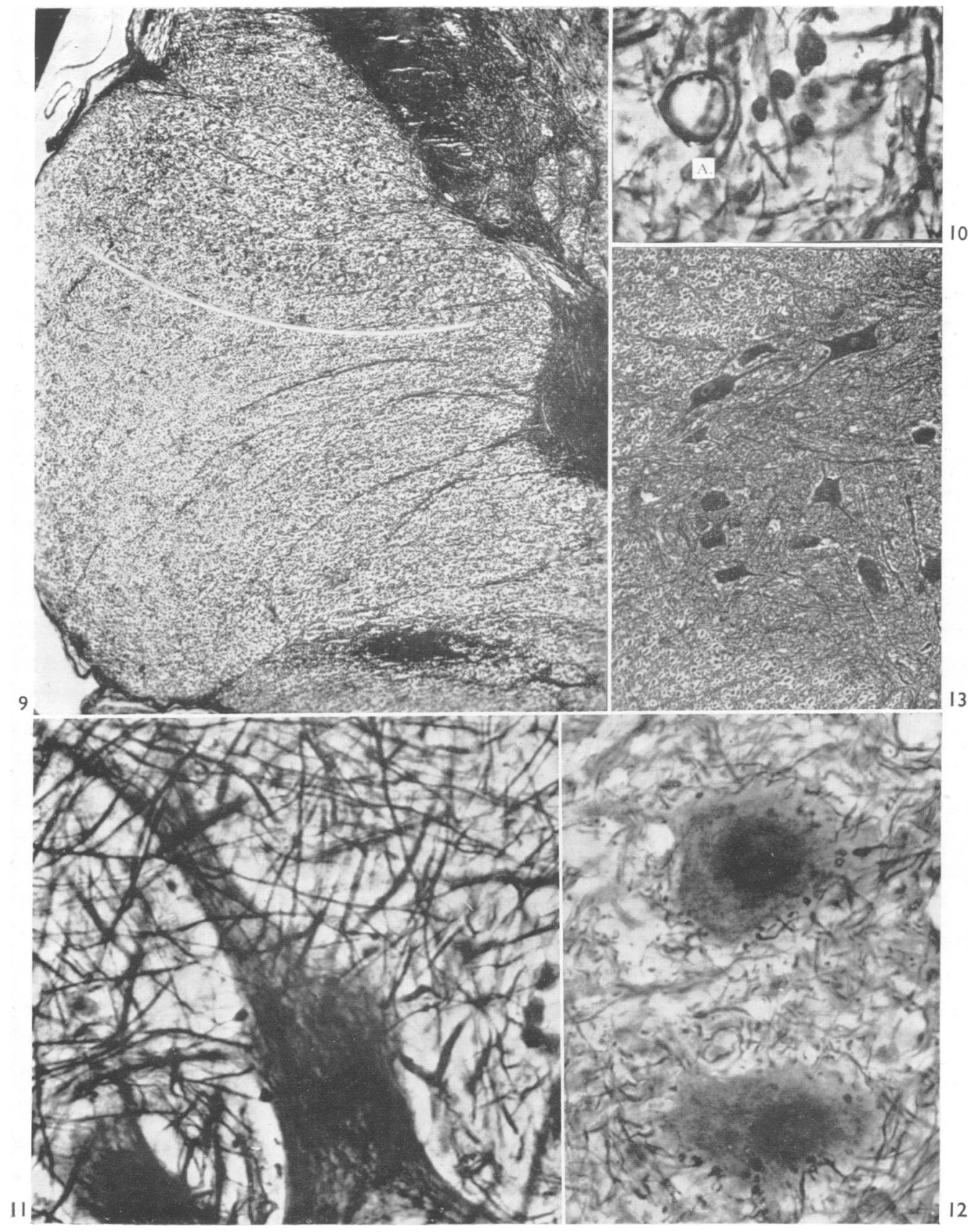

FIG. 9.-Degenerating myelinated ascending fibres (above white line) in the cuneate fascicle of the spinal cord of an adult cat, at the level of $C_{1}$. Fixed three days after removal of the right second spinal ganglion. Impregnation as in Fig. 2 . $(\times 53$.

FIG. 10.- Single degenerating myelinated fibre (above A) in the grey matter of the spinal cord of a cat, near to the level of $\mathrm{C}_{2}$. Fixing and impregnation as in Fig. 9. $(\times 650$.

Fig. 11.-Impregnation etc. as in Fig. 7 except that the albumen of the bath was taken from a $5 \%$ albumen stock solution preserved with thymol which was several months old. $(\times 850$.

Fig. 12.-Impregnation etc. as in Fig. 7 except the composition of the bath which was $0.2 \%$ albumin + $6 \% \mathrm{AgNO}_{3}$. Only fine, unmyelinated fibres, collaterals and end clubs impregnated. $(\times 650)$.

FIG. 13.- Multipolar nerve cells from the spinal cord of the same guinea-pig as Fig. 5. Fixation, decalcification the same as in Fig. $5(7 \mu)$. Direct protargol method. $(\times 100$. 
Slides are passed through benzene or xylene, and then, if celloidin-paraffin imbedding was used, through an ether-alcohol mixture to dissolve all traces of celloidin which would otherwise hinder impregnation, then through absolute alcohol, $95 \%$, and $70 \%$ alcohols into distilled water. Three changes in 5 to 10 minutes are sufficient, but several hours may give slightly clearer results. The washed slides are then put into the silver bath.

After fixing with "Susa" or other fixatives containing mercuric chloride, the slides have to be passed from the $96 \%$ alcohol into iodine-alcohol for the usual time to dissolve mercuric chloride, which interferes with impregnation. The slides are then put into $0.25 \%$ sodium thiosulphate solution to dissolve the mercuric iodate, and finally washed in distilled water, the routine practice with fixatives containing mercuric chloride.

Preparation of the Silver Colloid Bath.-It is advisable first to make a fresh $10 \%$ albumen stock solution, and then make up $100 \mathrm{ml}$. of $0.2 \%$ or $0.5 \%$ aqueous albumen solution by diluting some of the stock solution with the appropriate amount of distilled water. Finally crystallized silver nitrate, 2 g., is added to the solution and shaken until dissolved. Unless this procedure is followed, it is very inconvenient to measure the required small $(0.2$ or $0.5 \mathrm{ml}$.) quantities of the viscous, sticky egg albumen.

The egg albumen, if kept in glass and covered to avoid drying, can be used for making solutions for one to two weeks, and if dissolved as a $5 \%$ albumen solution in distilled water and a thymol crystal added for conservation, it can be used for many months ; it is very slightly less efficient than the undissolved fresh egg albumen (Fig. 11).

Dried egg albumen (purissimum) can be used as well as fresh egg albumen. The best procedure is to make a $0.1 \%$ stock solution of the dried product in distilled water (equivalent to $1 \%$ fresh egg albumen solution) and dilute from this for the bath. The dried egg albumen dissolves slowly. At room temperature at least one day is needed, after which some still remains as an undissolved sediment, but this can be neglected.

The addition of silver nitrate in stock solution (i.e. $10 \mathrm{ml}$. of $20 \%$ silver nitrate) to the egg albumen solution, instead of adding $2 \mathrm{~g}$. of crystallized silver nitrate, only works if the solution is freshly made. The older the silver nitrate solution, the less the selectivity of the impregnation; many days- or weeks-old silver nitrate stock solution gives a reddish, unselective stain to all tissues, probably because reduction of silver nitrate, with consequent nitric acid production, inevitably takes place in the solution with the passage of time.

Concentration of the Bath.-The concentration of the albumen or the silver nitrate and the albumen $\mathrm{AgNO}_{3}$ ratio seem to have no strict limits.

It is possible to impregnate, more or less satisfactorily, with other concentrations within a wide range, if the albumen is not lower than $0.02 \%$ and the $\mathrm{AgNO}_{3}$ is not lower than $\mathbf{0 . 7 5 \%}$. With lower percentages no satisfactory impregnation can be obtained with any change of the albumen $/ \mathrm{AgNO}_{3}$ ratio. The upper limit of the albumen and $\mathrm{AgNO}_{3}$ is not determinable because above $0.2 \%$ the albumen is already partly precipitated, and thus its concentration does not increase in the solution; a higher $\mathrm{AgNO}_{3}$ concentration than 2\% does not hinder or markedly improve the impregnation. A lower albumen concentration than $0.02 \%$ with any $\mathrm{AgNO}_{3}$ concentration gives a cloud-like diffuseness with fine granular impregnation in the neurocytes only but without neurofibrils, while the axons are not impregnated at all. $\mathrm{AgNO}_{3}$ with an albumen factor lower than $0.01 \%$ or without albumen usually does not impregnate; the tissues become a light reddish colour only.

If the albumen concentration is constantly set as low as possible for impregnation, i.e. $0.02 \%$, and the $\mathrm{AgNO}_{3}$ concentration is increased from $0.75 \%$ upwards, the impregnation is also shifted gradually from the perikaryon and dendrites to the finer, and later to the unmyelinated axons, till finally in a bath containing $0.02 \%$ albumen and $6 \% \mathrm{AgNO}_{3}$ only the thin unmyelinated collaterals and end clubs are selectively impregnated and appear almost black, while the thicker fibres are not distinct and appear reddish in colour, as do other tissues (Fig. 12). Unfortunately this reaction is not constant and vacillates as much as the old silver methods.

To sum up, a low $\mathrm{AgNO}_{3}$ concentration generally impregnates preferentially the neurofibrils of the perikaryon and its dendrites ; a higher $\mathrm{AgNO}_{3}$ concentration impregnates rather the unmyelinated fibres, collaterale and end clubs, in any albumen $/ \mathrm{AgNO}_{3}$ ratio. Since the higher and lower concentrations have disadvantages and give only specialized results, and the $0.2 \%$ or $0.5 \%$ albumen with $2 \% \mathrm{AgNO}_{2}$ formula gives usually the besw average results, it is suggested for general work.

Colour and Photosensitivity of the Bath.-The fresh silver colloid bath, containing $0 \cdot 2 \%$ albumen with $2 \%$ $\mathrm{AgNO}_{3}$ has a slight whitish-opalescent colour. It is photosensitive. If exposed for longer than a few minutes to direct sunlight or for a few hours to the daylight in a room, the bath turns a red-brown rusty colour (partly reduced silver colloid). This change should be prevented by placing the bath containing slides in the thermostat (incubator), or in a dark box if room temperature is preferred, because the partially reduced bath does not impregnate. For the same reason the bath must always be freshly made up.

Temperature of the Bath.-The temperature of the bath should be $37^{\circ} \mathrm{C}$. or less and certainly not above $38^{\circ}$ or $39^{\circ} \mathrm{C}$. Above $40^{\circ} \mathrm{C}$. the selectivity of the impregnation is perceptibly diminished, while above $45^{\circ} \mathrm{C}$. the axons are not impregnated, but other tissues, as well as the neurokeratin and other elements of the myelin sheaths, are impregnated. Room temperature seems to be better than any temperature above $37^{\circ} \mathrm{C}$.

Length of Impregnation.-The time required for $\overline{\mathrm{N}}$ impregnating is a function of the temperature. At $37^{\circ} \mathrm{C}$. $\sigma$ it is five days, and though good results can be obtained $N$ after three days or less, the results after five days are $\mathrm{N}$ always superior. At room temperature eight days is the $\sigma$ best impregnating period. After these periods the colour of the bath is rusty-brown. 
Gilding.-After impregnation in the bath the sections must be thoroughly washed with distilled water, for the albumen in the bath often hinders the effect of the gold. Then 3 to 6 drops of a $0 \cdot 25 \%$ gold chloride (yellow or brown) solution are dropped onto the horizontally placed slide, which is kept covered for 10 minutes. Gilding for seven minutes is certainly better than for three or five minutes, but a longer period than 10 minutes does not improve the results.

Reduction.-First wash off the gold by pouring distilled water on the slides for a few seconds, though prolonged washing even for a day does not alter the effect. Then the slides are placed for an hour in a $0.2 \%$ aqueous oxalic acid solution which reduces gold. In the oxalic acid solution the neurofibrillar substance appears within a few minutes. Reduction is usually nearly complete in seven to ten minutes, but it improves slightly for a further 30 to 50 minutes, after which time it is complete. If the sections are reduced for one day there is neither over-reduction nor any improvement.

The sections are dehydrated, cleared, and mounted in the usual way.

Results of Impregnation. - After chloral hydrate fixation slight shrinkage occurs because of the subsequent treatment in ammoniacal alcohol. The neurocytes and their dendrites are slightly violet ; both thick and thin axons and their collaterals, including end clubs, are black (Figs. 6, 7, 8). Nuclei are somewhat better impregnated than after fixation in ammoniacal alcohol. The granular disintegrating neurofibrillar substance of the degenerating axons are very strongly impregnated in black. The sheaths of the degenerating myelinated fibres are not shown.

After ammoniacal alcoholic fixation the tissues shrink markedly. The selectivity of the impregnation is greater. Under low power the neurocytes dominate the picture, because the neurofibrils of the perikaryon and dendrites are better preserved and impregnated than after other fixatives. Thick axons are grey, thinner ones are almost black (Fig. 1). Nuclei are not impregnated. Degenerating fibres appear the same as with the previous fixative. Sheaths of degenerating fibres do not appear.

After " Susa" fixation the selectivity is less than after the previous ones. Neurocytes, dendrites, and thicker axons have a lighter, more purple tone (Figs. 2, 3, 4). Nuclei are moderately impregnated. Degenerating axons, especially in the stage of granular disintegration of the neurofibrillar substance (Cajal's neurobions), are well impregnated, being somewhat darker and more violet in tone than the normal ones. The sheaths of the degenerated myelinated fibres appear remarkably distinct, because the surrounding of the myelin sheath is specifically impregnated while those of the non-degenerated myelinated fibres are invisible (Figs. 9, 10).

\section{The Direct Protargol (Gold) Method}

This technique differs from the direct albumen method only in the composition of the bath, which is a simple $2 \%$ protargol solution in distilled water without the addition of metallic copper or anything else. The results are very nearly the same as those given by the direct albumen method (Fig. 13).

\section{Indirect Albumen (Silver-Gold) Method}

This method is in fact only an auxiliary method to be used to complete the direct albumen method if sections have to be impregnated after other, less satisfactory fixatives which do not preserve the argyrophilia of the neurofibrillar substance as well as the three fixatives already suggested for use with the direct albumen silver method. Thus it may be used on sections of imbedded and stored blocks which have already been fixed in any fixatives excluding plain formalin or any other containing chromates, chromic or osmic acid, for these are entirely unsuitable for any of the three methods.

The indirect albumen method differs from the direct albumen method in the following respects.

First, the time in the bath is a little shorter. Secondly, washing after the bath is short and critical. Thirdly, there is the additional step of a reduction of silver before gilding. Fourthly, washing the slides after reduction in the silver bath has to be done thoroughly.

Fixation.-Any fixative can be tried except plain formalin and those containing chromates, chromic or osmic acid. Strongly acid fixatives (over $5 \%$ acid) are generally less satisfactory.

The techniques of imbedding, cutting, and preparing the silver colloid bath are the same as in the direct albumen method.

Reduction.-Imperfect control of the time of wash after the bath is a weak point in this and many other methods. If the wash is too long too much silver is removed, and the impregnation will not be complete enough ; if it is short, precipitate is left in the sections which will be patchy because the reducer cannot act well where some albumen has remained. Washing from 10 to 15 seconds with gently running distilled water seems to be optimal. Much depends on the fixative, however, and therefore sometimes several minutes of washing does not dissolve the bound and sensitized silver nucleus attached to the neurofibrillar substance.

The reducer is a sodium sulphite-sodium bisulphite, buffered, amidol solution with a $p \mathrm{H}$ of 6.5 to 7.5 which is the optimum for amidol (Davenport, McArthur, and Bruesch, 1939). We found this formula to be very superior to any other reducer, e.g., hydroquinone, pyrogallic acid, formol, etc., or their combinations. It is made up of five parts of stock aqueous $10 \%$ sodium sulphite and one part of aqueous $5 \%$ sodium bisulphite in $1 \%$ amidol. The latter has to be made fresh almost daily.

To perform the reduction, the reducing solution is dropped onto the slides and reduction is completed after 30 to 60 seconds.

Reduction is followed by washing of the slides, first with gently-running distilled water, and then in three changes of distilled water for one to three minutes. Washing has to be more complete than in the direct method, otherwise the remaining reducer interferes with gilding.

The techniques for gilding, the reduction of the gold, and dehydration, are the same as in the direct albumen method. 
Results of Impregnation.-These are similar to those of the direct albumen method, and any very unfavourable differences depend on the fixatives used and on bad luck during washing. The completeness of impregnation, however, never reaches the perfection of the direct albumen method.

\section{Reagents}

I have attempted to make all procedures in this study analytically clean, as is important in all impregnation methods, and I used analysed reagents, including benzene.

Absolute alcohol made by distillation, not by the addition of copper sulphate, has been used, because copper is one of the greatest enemies of silver impregnation except in Bodian's method. Alcohols of a lower percentage have been made by diluting distilled absolute alcohol with distilled water to eliminate the dirt which most alcohol acquires from its rusty iron containers. Glass-distilled water not older than three days was used, as metal-distilled or old glass-distilled water sometimes did not give the expected results.

Slides were not allowed to come into contact with metal forceps.

\section{Summary}

Three new methods are described for the impregnation of neurofibrillar substance in the central nervous system in sections prepared from imbedded material and then mounted on slides. The first of these, the direct albumen method, is considered simpler, more constant and uniform than any previous "on slide" method described.

\section{REFERENCE}

Davenport, H. A., McArthur, J., and Bruesch, S. R. (1939). Stain Tech., 14, 21. 